\title{
Squared-Off Nuclei and "Appliqué" Pattern as a Histopathological Clue to Periocular Sebaceous Carcinoma: A Clinicopathological Study of 50 Neoplasms From 46 Patients
}

Broekaert, Sigrid M C ; Flux, Katharina ; Kyrpychova, Liubov ; Kacerovska, Denisa ; Ivan, Doina ; Schön, Michael P ; Ströbel, Philipp ; Michal, Michal ; Denisjuk, Natalja ; Kerl, Katrin ; Kazakov, Dmitry V

DOI: https://doi.org/10.1097/DAD.0000000000000651

Posted at the Zurich Open Repository and Archive, University of Zurich ZORA URL: https://doi.org/10.5167/uzh-142500

Journal Article

Published Version

Originally published at:

Broekaert, Sigrid M C; Flux, Katharina; Kyrpychova, Liubov; Kacerovska, Denisa; Ivan, Doina; Schön, Michael P; Ströbel, Philipp; Michal, Michal; Denisjuk, Natalja; Kerl, Katrin; Kazakov, Dmitry V (2017). Squared-Off Nuclei and "Appliqué" Pattern as a Histopathological Clue to Periocular Sebaceous Carcinoma: A Clinicopathological Study of 50 Neoplasms From 46 Patients. American Journal of Dermatopathology, 39(4):275-278.

DOI: https://doi.org/10.1097/DAD.0000000000000651 


\title{
Squared-Off Nuclei and "Appliqué" Pattern as a Histopathological Clue to Periocular Sebaceous Carcinoma: A Clinicopathological Study of 50 Neoplasms From 46 Patients
}

\author{
Sigrid M. C. Broekaert, MD, * Katharina Flux, MD, † Liubov Kyrpychova, MD, $\neq$ \\ Denisa Kacerovska, MD, PhD, $\$$ Doina Ivan, MD, $\$ Michael P. Schön, MD, * Philipp Ströbel, MD, \| \\ Michal Michal, MD, $\neq$ Natalja Denisjuk, MD, ** Katrin Kerl, MD, †† and Dmitry V. Kazakov, MD, PhD $+\dagger$
}

\begin{abstract}
The histopathological diagnosis of periocular sebaceous carcinoma can be difficult in poorly differentiated cases showing few mature sebocytes. The authors examined 50 periocular sebaceous carcinomas from 46 patients to determine the frequency of 2 features seen in this neoplasm, namely cells with squared-off nuclei and so-called "appliqué" pattern (peritumoral subnecrosis of peripherally located neoplastic cells). Neoplastic cells with squared-off nuclei were found in varying numbers in both the intraepithelial and dermal (invasive) components in all neoplasms, whereas the appliqué pattern was observed in a third of the cases. It is concluded that these features, taken together, may serve as a clue for the diagnosis of periocular sebaceous carcinoma.
\end{abstract}

Key Words: sebaceous carcinoma, periocular, eyelid, sebaceous differentiation, squared-off nuclei, necrosis, appliqué pattern

(Am J Dermatopathol 2017;39:275-278)

\section{INTRODUCTION}

Periocular sebaceous carcinoma is a rare malignant tumor, usually affecting the elderly, mostly located on the eyelid, where most neoplasms originate from the meibomian gland. In contrast to its extraocular counterpart, periocular sebaceous carcinoma usually shows an aggressive behavior locally and metastasizes into regional lymph nodes and distant organs, most commonly the lung, liver,

From the *Department of Dermatology, Venereology and Allergology, University Medical Center Göttingen, Göttingen, Germany; †Dermatohistology and Oral Pathology Laboratory, Munich, Germany; \$Department of Pathology, Faculty of Medicine in Pilsen, Charles University in Prague, Pilsen, Czech Republic; §Bioptical Laboratory, Pilsen, Czech Republic; IDepartment of Pathology, University of Texas-MD Anderson Cancer Center, Houston, TX; \#Department of Pathology, University Medical Center Göttingen, Göttingen, Germany; **Dermatopathology Laboratory, Zurich, Switzerland; and ††Department of Dermatology, University Hospital, Zurich, Switzerland.

Supported in part by the Charles University Research Fund (project number SVV-2016-260 282).

The authors declare no conflicts of interest.

Reprints: Dmitry V. Kazakov, MD, PhD, Sikl's Department of Pathology, Charles University Medical Faculty Hospital, Alej Svobody 80, Pilsen 304 60, Czech Republic (e-mail: kazakov@medima.cz).

Copyright $(2017$ Wolters Kluwer Health, Inc. All rights reserved. bone, and brain. Periocular sebaceous carcinoma can simulate various inflammatory conditions clinically and may cause diagnostic problems histologically, as unequivocal sebaceous differentiation is often absent or difficult to recognize, especially in a limited biopsy specimen, often leading to an unfavorable delay of the final diagnosis. ${ }^{1-8}$

In our practice, we have often observed in periocular sebaceous carcinomas features resembling those seen in germ cell tumors (classical seminoma, embryonal carcinoma, or choriocarcinoma), namely "squared-off" or angulated nuclei and so-called "appliqué" pattern due to (sub)necrosis of peripherally located tumor cells in solid aggregations of neoplastic cells. ${ }^{9,10}$ The aforementioned features often served as a clue, forcing, when recognized, a meticulous search for areas with sebaceous differentiation in poorly differentiated neoplasms. The purpose of this study was to establish the frequency of squared-off nuclei and appliqué pattern in a large series of periocular sebaceous carcinoma.

\section{MATERIALS AND METHODS}

Fifty periocular sebaceous carcinomas from 46 patients were retrieved from our consultation, institutional, and personal files and reviewed to confirm the diagnosis and appropriate clinicopathological context. The clinical data were extracted from original pathological reports and referring pathologists. Only excisional biopsies of tumors were included; diagnostic biopsies were not studied. In 2 cases, the primary tumor and a recurrent neoplasm were available for review, and in another patient the primary tumor and 2 subsequent recurrences were analyzed. The study was based solely on light microscopy of hematoxylin and eosin-stained slides but prospectively, immunohistochemistry (epithelial membrane antigen and/or adipophilin) was performed in 32 cases, and additionally in 4 cases histochemistry (oil red O-staining) were carried out. We documented the presence of cells with squared-off nuclei in the intraepithelial (conjunctiva, epidermis, and sebaceous glands) and intradermal components and examined the peripheral parts of tumor aggregates to find peripheral (sub)necrosis of the neoplastic cells (appliqué pattern). 


\section{RESULTS}

\section{Clinical Data}

The cohort included 27 women and 17 men (gender was unknown in 2 cases), ranging in age at diagnosis from 24 to 92 years (median: 70 years). Locations included the upper eyelid $(\mathrm{n}=20)$, lower eyelid $(\mathrm{n}=14)$, eyelid, not specified $(\mathrm{n}=5)$, perirobital/periocular areas $(\mathrm{n}=3)$, caruncle $(\mathrm{n}=2)$, and conjunctiva $(\mathrm{n}=1)$. Additionally, in 3 patients, both the upper and lower eyelids were involved, 2 of whom presented with primary disease, and in the remaining patient involvement of both eyelids occurred during a second recurrence of the neoplasm that also extended into the orbita.

\section{Histopathological Findings}

Of the 50 neoplasms, 48 represented invasive carcinomas, whereas 2 were in situ lesions. The neoplasms were composed of small to large pleomorphic basaloid cells with
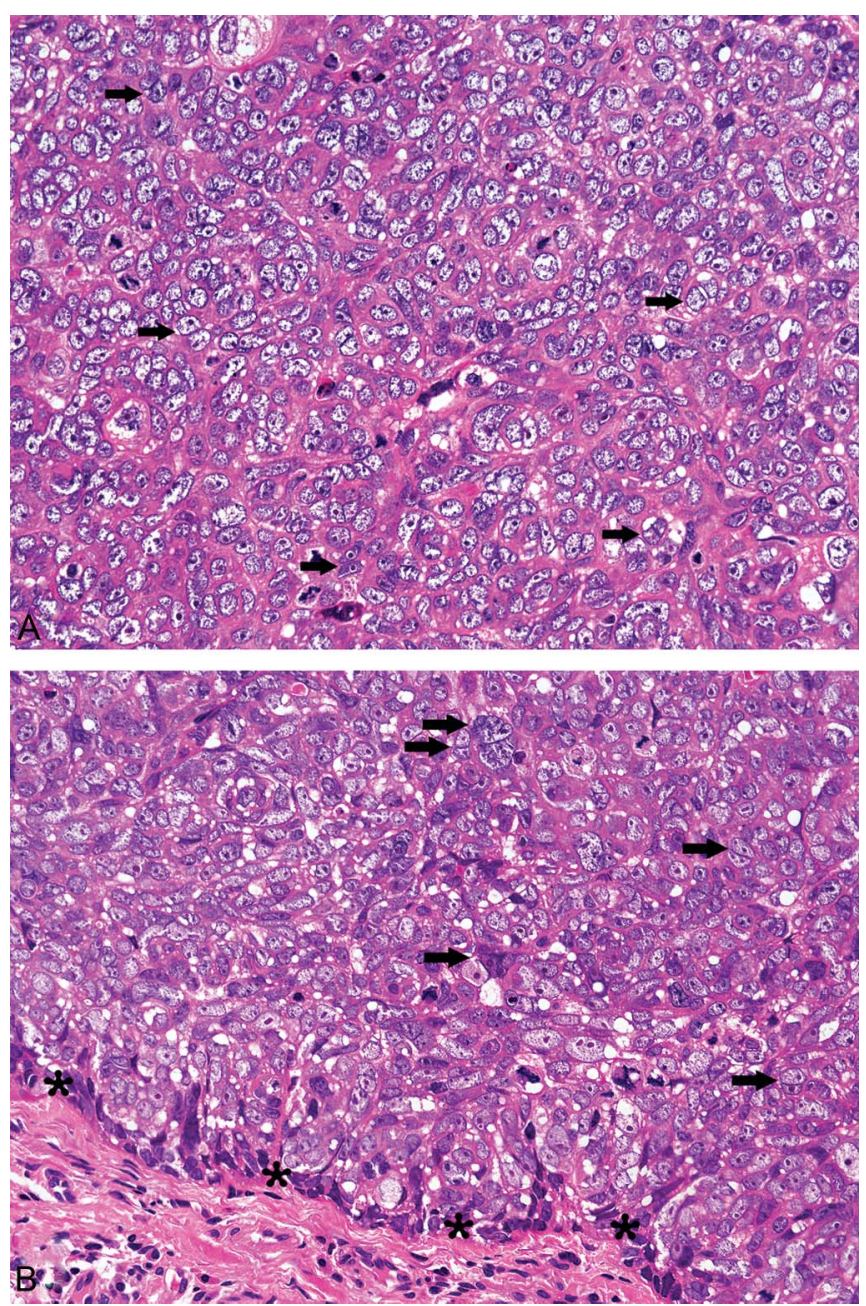

FIGURE 1. Periocular sebaceous carcinoma with neoplastic cells having pale vesicular nuclei with prominent nuclear membranes containing multiple squared-off/angulated nuclei (arrows) (A). The size of the nuclei varies and some cells are in close apposition (back to back, arrows) (B). Note also the peripheral cell necrosis (asterisks) (B). varying degrees of sebaceous differentiation identified by cells with multiple intracytoplasmic vacuoles with scalloped nuclei. Some lesions manifested rather pale vesicular nuclei. Marked nuclear pleomorphism, bizarre cells, and plentiful atypical mitoses were also seen.

Cells with squared-off or angulated nuclei were detected in all tumor specimens (100\%), either as single cells or lying in clusters, sometimes "back to back." The latter were usually seen in lesions with neoplastic cells having vesicular pale nuclei, in which the squared-off/angulated nuclei could generally be identified more easily (Figs. 1A, B). In tumors mostly composed of basaloid immature sebaceous cells, identification of cells with squared-off nuclei required a meticulous search (Fig. 2).

Of the 50 specimens, 42 cases contained epithelial parts (epidermis, conjunctival, or sebaceous glands), and the epithelial component was involved in 22 lesions $(52.4 \%)$, with all cases showing occasional neoplastic cells with squared-off nuclei. Overall, the intradermal (invasive) component contained more cells with squared-off nuclei compared with the intraepithelial component (Fig. 3). Cells with squared-off nuclei were usually either devoid of intracytoplasmic fat vacuoles or contained few of them or even a single intracytoplasmic vacuole (Fig. 4).

The appliqué pattern was identified in 16 of the 50 lesions $(32 \%)$, and this alteration was always present in the dermal invasive component of the neoplasms. The extent of this feature varied from only occasional neoplastic nodules with peripheral cell (sub)necrosis to rare lesions where most neoplastic cell aggregations displayed it (Figs. 1B, 5).

\section{DISCUSSION}

We have studied 50 tumors of periocular sebaceous carcinoma and found cells with squared-off nuclei in all cases, independent from the degree of sebaceous differentiation. The number of cells varied from case to case, and in tumors with pale vesicular nuclei and conspicuous nuclear

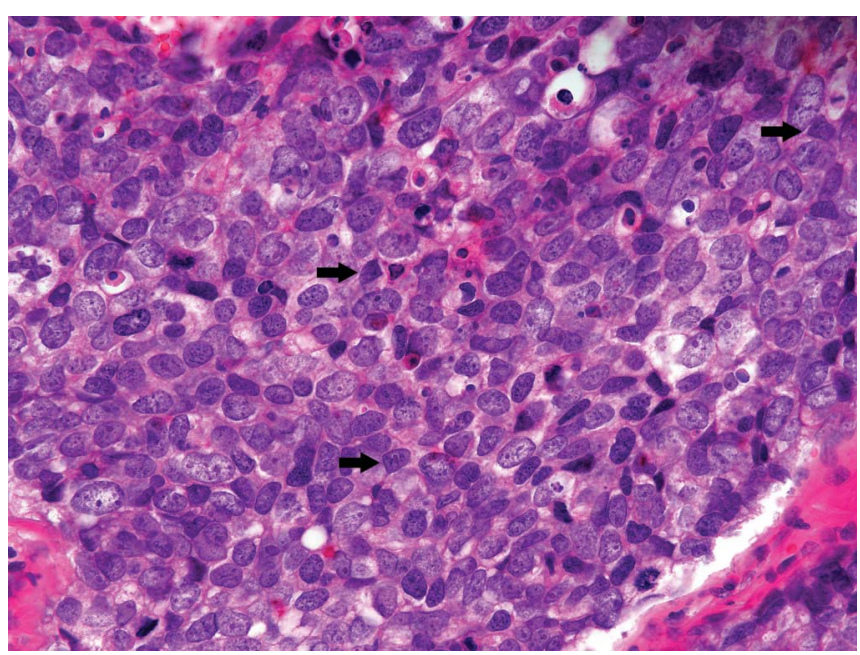

FIGURE 2. A neoplasm mainly composed of basaloid immature sebaceous cells. Cells with squared-off/angulated nuclei is more difficult to detect in such lesions.

Copyright (C) 2017 Wolters Kluwer Health, Inc. All rights reserved. 


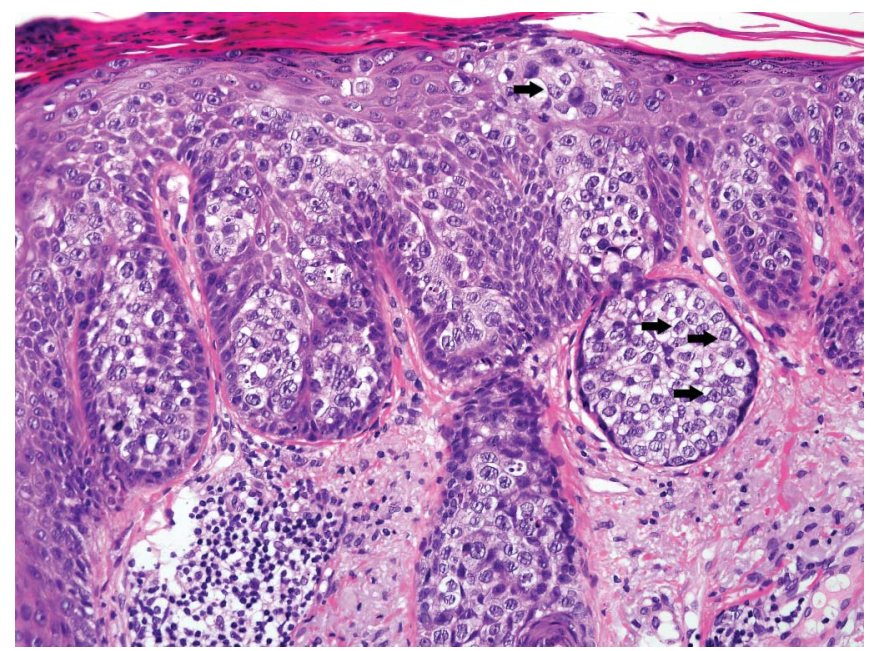

FIGURE 3. Sebaceous carcinoma with a prominent intraepithelial spread of neoplastic cells with numerous cells with squared-off nuclei (arrows).

membranes, the angulated shape was detected more easily, wherein clusters of neoplastic cells with squared-off nuclei occurred. These cells were present both in the invasive parts of the neoplasm and in the intraepithelial portions, albeit in the latter location they were a less conspicuous feature.

The appliqué pattern was found in a third of the cases examined (16 of 50 cases) and appeared to be less specific. Although we did not formally study a control group, we observed on occasion in the past peripheral cell (sub)necrosis in some other tumor types composed of basaloid cells, for example, in Merkel cell carcinoma. Taken together, however, we suggest that both features can serve as a quite characteristic, simple diagnostic clue for periocular sebaceous carcinomas, especially in a limited biopsy specimen where mature sebocytes may be absent, thus limiting also the usage of immunohistochemical staining with epithelial membrane antigen and adipophilin that label only mature sebaceous cells. In the

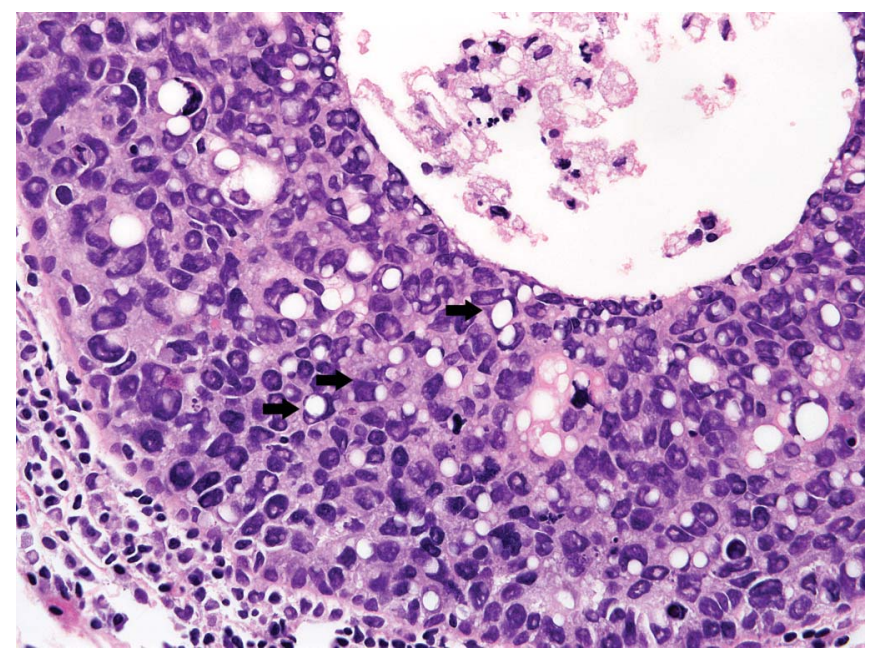

FIGURE 4. Cells with squared-off nuclei containing fat vacuoles (arrows).
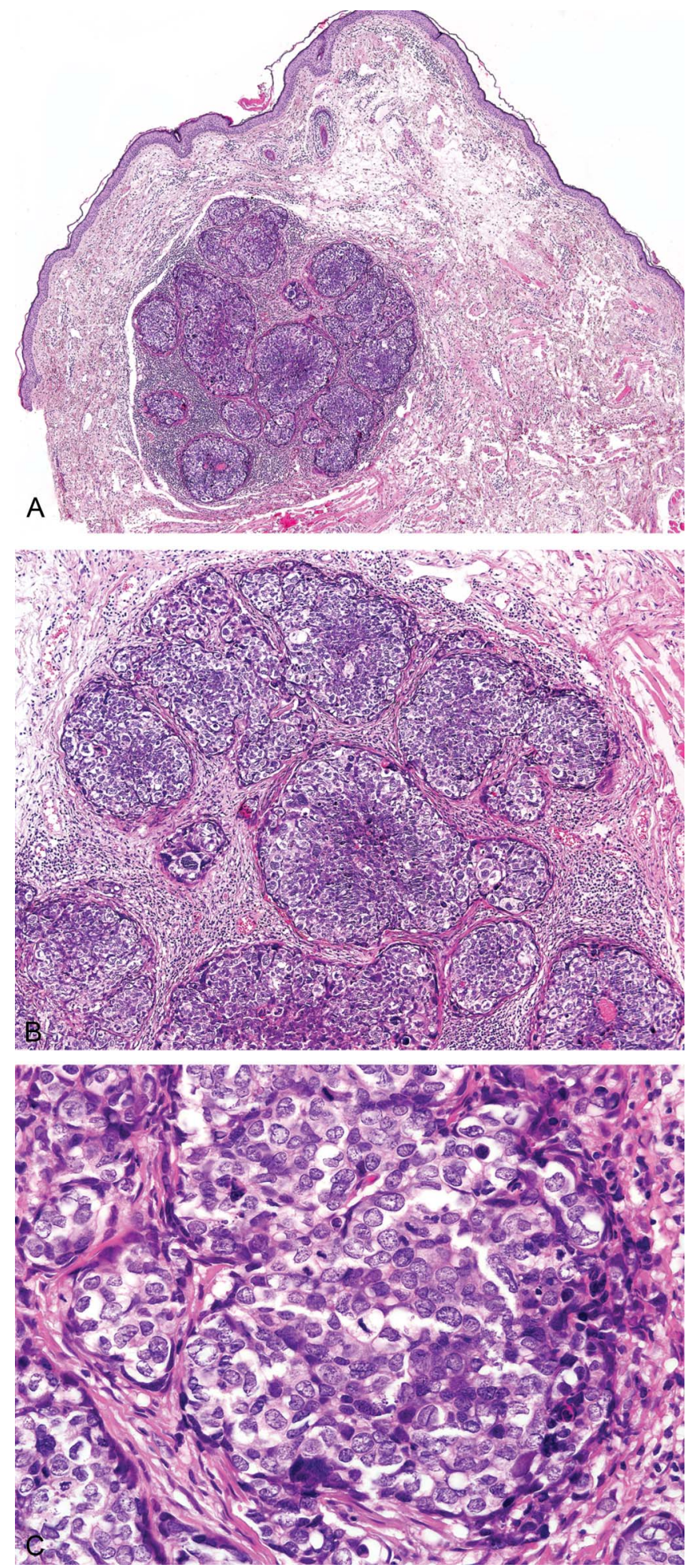

FIGURE 5. Periocular sebaceous carcinoma with the appliqué pattern seen in every tumor nodules (A and B). Note karyopyknosis and karyorrhexis of the neoplastic cells at the periphery of the neoplastic nodules (C). 
authors' experience, cells with squared-off nuclei are sometimes easier to identify on hematoxylin and eosin-stained slides than mature sebocytes with intracytoplasmic vacuoles.

In conclusion, neoplastic sebocytes with squared-off nuclei are a common feature in periocular sebaceous carcinomas, which can serve as a diagnostic clue. The appliqué pattern less common but seen in combination with squaredoff/angulated cells should prompt a search for signs of mature sebaceous differentiation in undifferentiated tumors located in the periocular area.

\section{REFERENCES}

1. Deprez M, Uffer S. Clinicopathological features of eyelid skin tumors. A retrospective study of 5504 cases and review of literature. Am J Dermatopathol. 2009;31:256-262.

2. Shields JA, Demirci H, Marr BP, et al. Sebaceous carcinoma of the eyelids: personal experience with 60 cases. Ophthalmology. 2004;111: $2151-2157$.
3. Shields JA, Demirci H, Marr BP, et al. Sebaceous carcinoma of the ocular region: a review. Surv Ophthalmol. 2005;50:103-122.

4. Honavar SG, Shields CL, Maus M, et al. Primary intraepithelial sebaceous gland carcinoma of the palpebral conjunctiva. Arch Ophthalmol. 2001;119:764-767.

5. Rao NA, Hidayat AA, McLean IW, et al. Sebaceous carcinomas of the ocular adnexa: a clinicopathologic study of 104 cases, with five-year follow-up data. Hum Pathol. 1982;13:113-122.

6. Ni C, Searl SS, Kuo PK, et al. Sebaceous cell carcinomas of the ocular adnexa. Int Ophthalmol Clin. 1982;22:23-61.

7. Jakobiec FA, Zimmerman LE, La Piana F, et al. Unusual eyelid tumors with sebaceous differentiation in the Muir-Torre syndrome. Rapid clinical regrowth and frank squamous transformation after biopsy. Ophthalmology. 1988;95:1543-1548.

8. Doxanas MT, Green WR. Sebaceous gland carcinoma. Review of 40 cases. Arch Ophthalmol. 1984;102:245-249.

9. Ulbright TM, Amin MB, Young RH. Tumors of the Testis, Adnexa, Spermatic Cord, and Scrotum. Washington, DC: AFIP; 1999.

10. Kazakov DV, Michal M, Kacerovska D, et al. Cutaneous Adnexal Tumors. Philadelphia, USA: LWW; 2012:814. 